\section{Toward Joint Assessments, Modern Capabilities, and New Links for Ocean Prediction Systems}

P. N. Vinayachandran, Fraser Davidson, and Eric P. Chassignet

\section{OceanPredict'19: Advancing the Science and Application of Ocean Predictions}

What: Approximately 260 individuals from forecasting centers, research laboratories, academia, and industry representing 40 countries met to discuss recent developments in operational oceanography and brainstorm about the future directions of ocean prediction services.

When: 6-10 May 2019

Where: Halifax, Nova Scotia, Canada

AFFILIATIONS: Vinayachandran-Centre for Atmospheric and Oceanic Sciences, Indian Institute of Science, Bangalore, India; Davidson-North Atlantic Fisheries Center, Oceanography Department, Fisheries and Oceans Canada, St. John's, Newfoundland and Labrador, Canada; Chassignet-Center for OceanAtmospheric Prediction Studies, Florida State University, Tallahassee, Florida

https://doi.org/10.1175/BAMS-D-19-0276.1

Corresponding author: P. N. Vinayachandran, vinay@iisc.ac.in

In final form 6 September 2019

For information regarding reuse of this content and general copyright information, consult the AMS Copyright Policy. 
$\mathrm{F}$ or the past two decades, operational agencies from different nations have provided oceanography services using ocean prediction systems. These forecasts have found applications in marine activity areas such as safety of life at sea, water quality and pollution monitoring, coastal regions management, fisheries and aquaculture, ocean science, and climate research. Essential components of any ocean prediction system are observing systems, data management systems, numerical models, data assimilation schemes, and forecast dissemination mechanisms. These components evolve continuously, requiring effort in upkeep and improvements that lead to increased forecast accuracy and quality. In spring 2019, OceanPredict'19 provided a forum to discuss recent developments in operational oceanography and brainstormed on future directions of ocean prediction services. The overarching goals of OceanPredict'19 were to

- build a common picture of the future of operational oceanography,

- motivate integrated international community projects,

- enhance end-user awareness of present ocean prediction capacity, and

- provide collaborative outputs for end-user engagement.

OceanPredict'19 was organized by GODAE OceanView (now OceanPredict). The vision for GODAE (Global Ocean Data Assimilation Experiment), formulated in 1997, was to be a "global system of observations, communications, modelling and assimilation, that will deliver regular, comprehensive information on the state of the oceans, in a way that will promote and engender wide utility and availability of this resource for maximum benefit to the community.” GODAE transitioned to GODAE OceanView in 2009 to define, monitor, and promote actions aimed at coordinating and integrating research associated with multiscale and multidisciplinary ocean analysis and forecasting systems. In 2019, GODAE OceanView was renamed OceanPredict to emphasize ocean prediction as part of the broader network of international operational oceanography initiatives that also include observing systems, data management, and information service delivery.

Several recommendations emerged from the plenaries, splinter sessions, poster sessions, and town hall discussions. First and foremost is that the ocean is only one component of the Earth prediction system and that there must be careful evaluation of how the various components interact and impact each other. This is especially true for atmospheric forcing, which is prescribed in most short-term ocean forecasting systems. There are significant efforts being put into developing seamless coupled ocean-ice-atmospheric forecasting systems from 1 to 90 days out (U.S. Navy ESPC, U.S. NOAA EPIC, Met Office, ECMWF, etc.). The current crop of ocean models, however, do not allow for any ocean-atmosphere feedback when computing the wind stress, a significant omission considering that it has been shown to meaningfully impact the representation of western boundary currents via the socalled eddy-killing effect. Considerable progress has also been made in the development of high-resolution ocean models, with significant improvements in western boundary current pathways when submesoscale eddies are resolved. Along these lines, the symposium also drew attention to the impact of higher-order numerical schemes and unstructured grids on improving the accuracy of ocean model simulations. Another critical component of the Earth system is biogeochemistry. Prediction of marine biogeochemical parameters has a variety of scientific and societal applications including carbon cycle and fisheries management, yet forecasting of biogeochemical variables is in its infancy. Most of the large-scale coupled physical-biological models are highly nonlinear and have many poorly known parameters that are not properly validated, primarily due to the lack of appropriate datasets. Accordingly, models vary greatly among themselves in forecasting primary productivity. Systematic and regular data collection is an immediate requirement for forecasting systems. It is expected 
that the recent development of Argo floats with biogeochemical sensors will contribute significantly to these efforts in the coming years.

Second, because sustained ocean observations are a basic requirement for ocean prediction, the OceanPredict community needs to continuously engage with the observational community to provide impact statements on the various observation systems and how they influence assessment and predictions. Ocean observations play a critical role in all aspects of operational oceanography, not only for assimilation but as part of the research cycle and for verification and validation of products. At present, most deep ocean prediction systems assimilate satellite sea level anomalies and sea surface temperature, complemented by in situ temperature and salinity profiles. A key observing system for in situ profiles is the Argo array-a global array of free-drifting profiling floats $(4,000)$ that provides $\sim 14,000$ profiles each year of temperature and salinity in the upper 2,000 $\mathrm{m}$ of the ocean. Soon Argo will provide deeper profiles as well as other observation types (biogeochemistry). Observations are more limited in shelf seas, and this was recognized as a challenge. The real-time and delayed mode observations needed for operational oceanography mean that there must be two versions of each observation: one with automated, robust quality control that is available for use in real-time, and the other with the best available quality control, often including manual intervention, but available with a longer delay that should offer improved accuracy. To ensure traceability, a data management system capable of managing both options is required. From an operational oceanography perspective, it is critical to have metadata that clearly flag what quality control has been undertaken and the error characteristics of each observation.

Third, it was agreed that the major challenges faced by present-day data assimilation schemes are in quantifying errors of both observations and models and developing appropriate schemes for dealing with submesoscale features, taking advantage of biogeochemistry measurements. It is also likely that we will see improvements in ensemble/variational hybrid data assimilation and coupled data assimilation that could capitalize on community resources fostered by the OceanPredict community. Verification of ocean predictions has gradually evolved using metrics defined as sets of diagnostics that compute scalar measures from ocean forecasting systems outcomes, providing not only objective quality indicators that can be quantitatively compared but also uncertainty estimates and error. With the motivation toward much higher horizontal resolution in the prediction systems, these traditional metrics may be unable to demonstrate an improvement in forecast skill. While higher-resolution models can generate more realistic fields, it is often the case that statistics based on direct point match-ups between interpolated models and observations do not improve. There is, therefore, a need to develop more sophisticated neighborhood methods and ad hoc metrics to deliver more accurate assessments of forecast skill at the local level.

Finally, there was consensus that the major challenges likely to be faced by future ocean prediction systems include constructing a global infrastructure for sharing data from both observations and models, as well as best practices. Hazards originating out of natural or man-made causes are likely to be more and more impactful in the future; thus, prediction systems are likely to be challenged for their preparedness and resilience. Increasing ocean literacy and improved communication among the observation and forecasting communities needs to go hand-in-hand with scientific and technological advances. The United Nations Decade of Ocean Science for Sustainable Development (2021-30) is an opportunity to build new partnerships and to develop a comprehensive observing system. We cannot anticipate all of the future uses that will be made of ocean forecasts systems, but it is likely that these end uses will evolve alongside the evolution (increased skill at longer lead times and a wider range of simulated parameters) of the prediction systems themselves, as well as with better accessibility tools and application program interfaces. 